\title{
Meningovascular syphilis of the spinal cord presenting with incomplete Brown-Séquard syndrome:
}

\section{case report}

\author{
P M TERRY,* G R GLANCY, $\dagger$ A GRAHAM $\ddagger$
}

From the *Department of Genitourinary Medicine, Praed Street Clinic, St Mary's Hospital, and the $\dagger$ Department of General Medicine, St Charles's Hospital, London, and the $\ddagger$ National Spinal Injuries Centre, Stoke Mandeville Hospital, Aylesbury, Buckinghamshire

SUMMARY A man aged 31 presented to hospital with acute onset of weakness in the legs, sensory loss, and disturbance of bladder and bowel function. Incomplete Brown-Séquard syndrome secondary to meningovascular syphilis of the spinal cord was diagnosed after serological tests for syphilis gave positive results. His condition was probably caused by endarteritis of the arteria radicularis magna of Adamkiewicz. He was treated with procaine penicillin 1.8 MIU intramuscularly once a day and probenecid $0.5 \mathrm{~g}$ by mouth three times a day for 21 days followed by physiotherapy and rehabilitation. Considerable neurological recovery was expected. To our knowledge this association has not been reported previously in detail in an English publication.

\section{Case report}

A single Portuguese man aged 31 presented to the accident and emergency department of St Charles's Hospital giving a 24 hour history of weakness in the left leg. On initial examination he was apyrexial, his pulse rate was 60 beats/minute in sinus rhythm, blood pressure was $120 / 75 \mathrm{~mm} \mathrm{Hg}$, and his respiratory rate was 20 breaths/minute. His mental state and results of cranial nerve examinations showed no abnormality apart from slight pallor of the right optic disc. His muscle tone was normal, but all muscle groups in his left leg were weak. All tendon reflexes were present, but were increased in the left leg. Both plantar responses were equivocal, and no sensory disturbance was noted. His condition deteriorated during five days after admission to hospital, with complete loss of voluntary movement in the left leg, loss of bladder and bowel sensation, faecal incontinence, and urine retention with overflow incontinence. Weakness developed in all muscle groups of the right leg and increased muscle tone, hyperreflexia, and bilateral extensor plantar responses were noted. Abdominal reflexes were absent, but the anal reflex was preserved.

Address for reprints: Dr P M Terry, Department of Genitourinary Medicine, Royal South Hants Hospital, Graham Road, Southampton, Hampshire SO9 4PE

Accepted for publication 17 January 1989
Appreciation of pin prick and temperature was now impaired in the right leg and trunk to the T5 level, anteriorly and posteriorly, but remained normal in the left leg and trunk. Appreciation of light touch was impaired in the left leg and trunk to the L1 level. Vibration sense was impaired bilaterally below the level of the rib cage, and joint position sense was impaired, but not lost, in both feet.

Investigations of full blood count, serum urea and electrolyte concentrations, and liver function gave normal results. Random blood glucose concentration was $5.6 \mathrm{mmol} / \mathrm{l}$. Chest radiography and electrocardiography showed no abnormalities. A myelogram showed no abnormality up to the level of the foramen magnum, and visual evoked responses were normal. Repeat serological testing gave positive rapid plasma reagin (RPR) test results at a titre of $1 / 128$, and the Treponema pallidum haemagglutination assay (TPHA) and fluorescent treponemal antibody absorption (FTA-ABS) test also gave positive results. Lumbar puncture was performed, and clear cerebrospinal fluid (CSF) obtained. Analysis of the CSF showed $60 \times 10^{6}$ white blood cells $/ 1(100 \%$ lymphocytes) and $10 \times 10^{6}$ red blood cells/1. Auramine and Gram stained specimens of the CSF showed no organisms, and no bacterial growth was obtained. CSF protein concentration was $2.0 \mathrm{~g} / 1$ (normal range $0.1-0.4)$ and CSF glucose concentration $1.8 \mathrm{mmol} / \mathrm{l}$. TPHA and FTA-ABS tests of the CSF gave positive results and the RPR test, having given a negative result 
initially, was positive at a titre of $1 / 32$ when repeated six weeks after the patient's admission to hospital.

The provisional clinical diagnosis was that of multiple sclerosis, and treatment with dexamethasone $4 \mathrm{mg}$ three times a day was started. The results of serological tests for syphilis, however, suggested a diagnosis of meningovascular syphilis affecting the spinal cord. Treatment with dexamethasone was therefore withdrawn and substituted with daily intramuscular injections of procaine penicillin $1.8 \mathrm{MIU}$, and probenecid $0.5 \mathrm{~g}$ by mouth three times a day, for 21 days. At this stage the patient was noted to be a bisexual man whose last sexual contact (homosexual) had occurred three months previously. No evidence of human immunodeficiency virus (HIV) infection was found on serological testing (Wellcozyme; Wellcome Laboratories) performed after obtaining his informed consent.

The patient initially remained confined to bed or a wheelchair and required an indwelling urinary catheter, regular bowel enemas, and antispasmodic drugs. Regular physiotherapy was arranged, and two months after admission to hospital he was transferred to the spinal injuries unit at Stoke Mandeville Hospital for further rehabilitation. Six months after treatment he was able to stand and walk a few paces with crutches and a long leg caliper on the left leg. Bladder and bowel sensation remained absent, and intermittent bladder catheterisation and daily manual evacuation of the rectum were required. Nine months after treatment mobility had improved further, the bladder emptied reflexly, but manual evacuation of the rectum remained necessary.

The serum VDRL test result was positive at titres of $1 / 3275$ days after treatment and of $1 / 16$ after 186 days. Nine months after treatment the CSF VDRL test result was negative.

\section{Discussion}

The initial clinical diagnosis for this patient was incomplete Brown-Séquard syndrome, and multiple sclerosis was considered to be the most likely cause. Finding positive results to serological tests for syphilis in both the serum and CSF, however, with high protein concentrations and lymphocytic pleocytosis in the CSF, suggested the diagnosis of meningovascular syphilis of the spinal cord. Stokes et al referred to the Brown-Séquard syndrome occurring as a result of gummas of the spinal cord, ${ }^{1}$ and Cole reported it resulting from "acute syphilitic transverse myelitis"." Adams and Merritt also quoted 34 cases of the BrownSéquard syndrome reported by Petrén, ${ }^{3}$ of which "several were due to spinal syphilis." 4 To our knowledge, however, the association of meningovascular syphilis and the Brown-Séquard syndrome has not been reported previously in detail in an English publication.

Syphilis of the spinal cord is a rare disorder. Adams and Merritt reported 31 cases of spinal infection in 2231 patients with syphilis seen at the Boston City Hospital during the era before antibiotics. ${ }^{4}$ After the introduction of antibiotics, a review by Hotson found "acute or chronic myelopathy" in 5\% of patients presenting with neurosyphilis between 1965 and 1980, and Wolters reported an incidence of "spinal meningovasculitis" in $5 \%$ of 121 patients diagnosed as having neurosyphilis in Amsterdam between 1970 and $1984 .^{\circ}$ Adams and Merritt suggested that the basic pathological lesion in spinal syphilis is spinal meningitis that, when chronic, leads to degeneration of both dorsal and ventral nerve roots or peripherally placed fibres of the spinal cord (meningomyelitis). ${ }^{4}$ The occurrence of endarteritis, however, leads to spinal cord infarction (myelomalacia) that is characterised by rapid onset of symptoms and signs, in contrast to meningomyelitis, and was the most likely diagnosis in this case.

The blood supply of the spinal cord is derived from anterior and posterior spinal arteries and the radicular branches of several segmental arteries, which together form longitudinal anastomoses along the cord. ${ }^{7}$ The most important of these radicular arteries is the arteria radicularis magna of Adamkiewicz, which arises from a branch of the descending aorta in the lower thoracic or upper lumbar vertebral levels. It may be responsible for the blood supply of as much as the lower two thirds of the spinal cord, and was probably the artery affected in this case.

An added current dimension to managing patients with syphilis is the possibility of concurrent infection with HIV. Johns et al reported four cases of neurosyphilis in young homosexual men with serological evidence of exposure to HIV and suggested that HIV infection may have modified the natural history of syphilis in these patients by increasing the propensity of the disease to progress to neurosyphilis, by decreasing the period of latency before the onset of neurosyphilis, and by increasing the severity of manifestations of neurosyphilis. ${ }^{8}$ Underlying HIV infection was considered to be a distinct possibility in our patient because of his sexual orientation and the severity of his disease, but he proved not to be infected.

Some recovery of neurological function may be expected after ischaemia of the spinal cord. Silver and Buxton studied 11 patients who presented with flaccid paralysis and absent tendon reflexes in the legs, with sensory loss, as a result of a fall in the perfusion pressure of the spinal vessels. ${ }^{9}$ They noted considerable neurological recovery in motor power and appreciation of sensation, but they commented that the absence of major histological changes on necropsy 
of two patients may explain neurological recovery in many cases. Garland et al reported a similar case after coronary thrombosis and a case of spinal ischaemia resulting from the ligation, at operation, of intercostal arteries. ${ }^{10}$ In both cases, considerable neurological recovery ensued.

Syphilis is rarely seen in the United Kingdom outside clinics for sexually transmitted diseases and may not be considered in a differential diagnosis, particularly if a patient's sexual orientation is not known. This man presented to an accident and emergency department and was admitted under a general medical firm, which shows the value of requesting serological tests for syphilis routinely in patients presenting with acute neurological symptoms and signs.

We thank Drs J H Baron, J R Silver, and E A Nieman for permission to report this case and for their helpful advice.

\section{Refereaces}

1 Stokes JH, Beerman H, Ingraham NR. Late syphilis of the nervous system. In: Modern clinical syphilology. Philadelphia: WB Saunders, 1944:967-1067.

2 Cole HN. Acute syphilitic transverse myelitis. Archives of Dermatology and Syphilology 1924;9:102-13.

3 Petrén. Ein beitrag zur frage nach dem verlaufe der bahnen der hautsinne in ruchenmark. Skandinavisches Arch für Physiologie 1902;13:9-98 (cited in next reference.)

4 Adams RD, Merritt HH. Meningeal and vascular syphilis of the spinal cord. Medicine (Baltimore) 1944;23:181-214.

5 Hotson JR. Modern neurosyphilis: a partially treated chronic meningitis. West J Med 1981;135:191-200.

6 Wolters ECh. Neurosyphilis: a changing diagnostic problem? Eur Neurol 1987;26:23-8.

7 Williams PL, Warwick R, eds. Neurology. In: Gray's anatomy. Edinburgh: Churchill Livingstone, 1980:802-1226.

8 Johns DR, Tierney M, Felsenstein D. Alteration in the natural history of neurosyphilis by concurrent infection with the human immunodeficiency virus. $N$ Engl J Med 1987;316:1569-72.

9 Silver JR, Buxton PH. Spinal stroke. Brain 1974;97:539-50.

10 Garland H, Greenberg J, Harriman DGF. Infarction of the spinal cord. Brain 1966;89:645-62. 